PREPARED FOR SUBMisSion to JHEP

\title{
Di-Higgs Production in SUSY models at the LHC
}

\author{
Peisi Huang, and Yu Hang Ng \\ Department of Physics and Astronomy, University of Nebraska-Lincoln, Lincoln, NE, 68588 \\ E-mail: peisi.huang@unl.edu, yu-hang.ng@huskers.unl.edu
}

ABSTRACT: We study the modification to di-Higgs production via gluon fusion within the context of the Minimal Supersymmetric Standard Model(MSSM) and the Next-toMinimal Supersymmetric Standard Model(NMSSM) in the parameter space allowed by current experimental and theoretical constraints, and also relevant to the Large Hadron Collider(LHC) experiments in the near future. The calculation is based on the analytical expression of the leading order Feynman amplitudes (which includes both quark and squark loops). We separate the di-Higgs production cross section into resonant, non-resonant, and interference parts, in order to better understand the mechanisms that are responsible for the modification to di-Higgs production rate in different regions of the allowed parameter space. We also investigate the sensitivity of High-Luminosity LHC (HL-LHC) to the di-Higgs production in these low energy supersymmetry(SUSY) models. Furthermore, we examine the complementarity between di-Higgs searches and direct searches for BSM particles and precision Higgs couplings measurements at the HL-LHC. We found that the di-Higgs production cross section can be enhanced significantly through resonant production. In the region where the resonant production cross section is small, di-Higgs receives a moderate enhancement due to the modifications in the Higgs couplings. In addition, there is a strong correlation between di-Higgs production and single Higgs production, and di-Higgs is not as sensitive as single Higgs at the HL-LHC. 


\section{Contents}

1 Introduction 1

2 Matrix Elements and Cross Sections 2

2.1 MSSM 2

2.2 NMSSM 5

3 Phenomenological Study 5

3.1 MSSM 5

$\begin{array}{lll}3.2 & \text { NMSSM } & 9\end{array}$

4 Conclusions $\quad 11$

A Form Factors 13

\section{Introduction}

The Higgs boson discovery by the CMS and ATLAS experiments in $2012[1,2]$ is the first and a crucial step in understanding the mechanism of the electroweak symmetry breaking. The Higgs properties, including the mass, spin, parity, and its couplings to other Standard Model (SM) particles have been measured with precisions in the subsequent analysis [3-6]. However, we know very little about the Higgs potential. In the SM, the Higgs potential is

$$
V=-\mu^{2} \phi^{\dagger} \phi+\lambda\left(\phi^{\dagger} \phi\right)^{2},
$$

which is completely specified by two parameters $\mu$ and $\lambda . \mu$ and $\lambda$ can be determined from the vacuum expectation value (vev) of the Higgs field, and the mass of the Higgs boson, but there is no direct measurement beyond that. Thus, the next step in understanding the shape of the Higgs potential is to measure the Higgs trilinear coupling. The Higgs trilinear coupling can be probed by the di-Higgs production at the LHC.

Di-Higgs production is sensitive to new physics, including new scalar resonances [7-10], new colored particles [10-13], and modified Higgs couplings [8, 10, 13, 14], and therefore can be complementary to direct searches of new particles, and precision Higgs coupling measurements. In particular, in this paper, we consider di-Higgs production with low energy supersymmetry (SUSY) models.

In low energy SUSY models, the di-Higgs production rate can be modified through various mechanisms. First, the Higgs sector is extended by introducing an additional Higgs doublet, in the Minimal Supersymmetric extension of the SM (MSSM) and an additional singlet in the Next-to-Minimal Supersymmetric extension of the SM (NMSSM). The additional neutral Higgs states can be produced at the LHC and can decay into a pair of 
SM-like Higgs bosons, and therefore contribute to di-Higgs production. Second, the low energy SUSY models allow the presence of new light colored particles coupled strongly to the Higgs. Those new colored particles give new QCD loop diagrams contributing to di-Higgs production $[11,13]$. There will be new interference terms arise from those new diagrams as well $[15,16]$. Third, in SUSY models, Higgs couplings, including Higgs couplings to SM particles, and the Higgs self-couplings, may present small deviations with respect to the SM ones, resulting in modified di-Higgs production [13]. The modified couplings also change the decay of the Higgs, resulting in additional modifications in specific channels. In this paper, we study the di-Higgs production in the MSSM and the NMSSM, with a focus on identifying the dominant contribution in different regions of parameter space. We also study the complementarity between di-Higgs searches and other searches, such as direct searches for new scalars, and precision Higgs measurements at the HL-LHC.

This article is structured as follows. In section 2, we calculate the leading order diHiggs production cross section in the MSSM and the NMSSM. In section 3, we present the main results of this paper. First, we identify the parameter space that satisfies current experimental constraints and theoretical requirements. Then, we show the results of di-Higgs production cross section in the $b \bar{b} \tau^{+} \tau^{-}$and $b \bar{b} \gamma \gamma$ final states, in those regions. Furthermore, we study the complementarity of di-Higgs with other searches. We also discuss the resonant, non-resonant, and interference contributions of the di-Higgs production in different regions of the parameter space. Finally, we reserve section 4 for a summary of the main results.

\section{Matrix Elements and Cross Sections}

The dominant di-Higgs production mode at the LHC is the gluon-gluon fusion (ggF) process. In the SM, there are two diagrams contributing to the di-Higgs production at the leading order, the triangle diagram (diagram (1) in Fig. 1, with $H_{i}=h$ ), and the box diagram (diagram (2) in Fig. 1). In this section, we calculate the leading order di-Higgs production cross section $\sigma(p p \rightarrow g g \rightarrow h h)$ at $\sqrt{s}=14 \mathrm{TeV}$ by using the analytical expressions for one-loop amplitudes of $g g \rightarrow h h$ in the MSSM and the NMSSM.

\section{$2.1 \quad$ MSSM}

The leading order Feynman diagrams contributing to the $g g \rightarrow h h$ process in the MSSM at the LHC are summarized in Fig. 1. In the MSSM, there are two neutral CP-even Higgs bosons and one neutral CP-odd Higgs boson. In this work, we assume that the lighter CP-even neutral Higgs boson to be SM-like, and denote the SM-like Higgs boson as $h$, and the heavy CP-even neutral heavy Higgs boson as $H$. The heavy Higgs can be produced through quark and squark loops and can decay to a pair of SM Higgs bosons, as shown in diagrams (1), (3), and (4) in Fig. 1 with $H_{i}=H$. The squarks also lead to new diagrams that contribute to di-Higgs production, as shown in diagrams (3)-(8) in Fig. 1. The corresponding spin and color averaged partonic differential cross section is

$$
\frac{d \hat{\sigma}}{d \hat{t}}=\frac{\left(\alpha_{s}(Q)\right)^{2}}{2^{13} \pi^{3}}\left(|A|^{2}+|B|^{2}\right),
$$




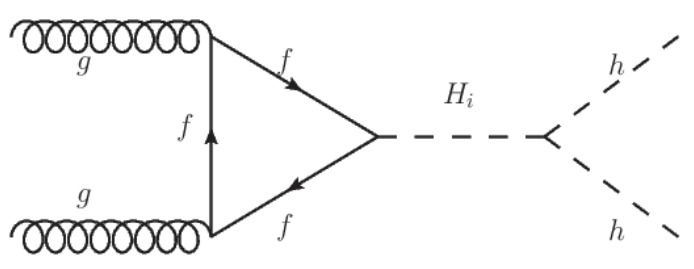

(1)

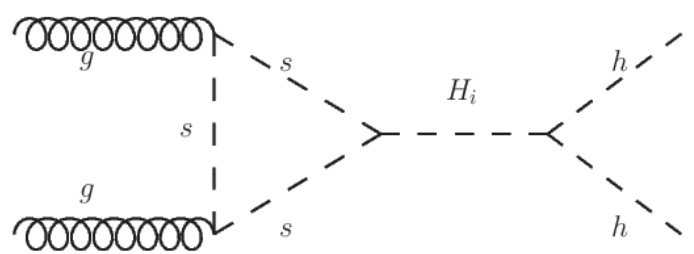

(3)

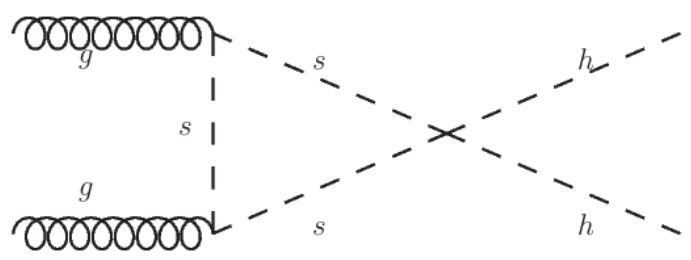

$(5)$

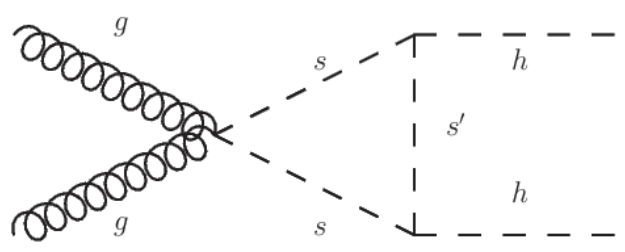

(7)

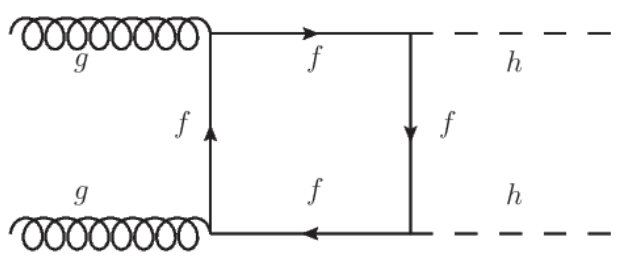

(2)

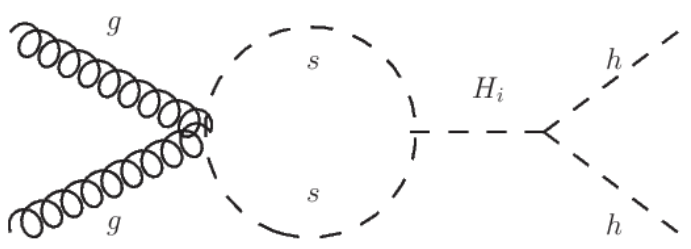

(4)

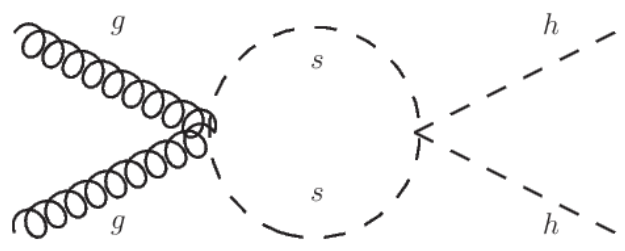

(6)

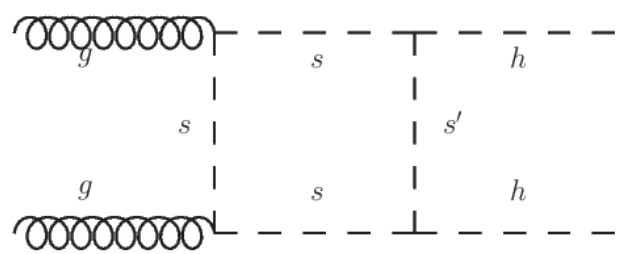

(8)

Figure 1. Leading order Feynman diagrams for di-Higgs production in the MSSM at LHC. $H_{i}$ are CP even Higgs bosons, $f$ are top and bottom quarks, and $s, s^{\prime}$ are stops.

where the matrix elements are separated by the helicities of the initial gluons. $A_{(i)}$ are matrix elements of the $i$-th diagram in Fig. 1 when helicities of initial gluons are identical, and $B_{(i)}$ are matrix elements of the $i$-th diagram in Fig.1 when helicities of initial gluons are opposite. A and B denote the sum of those matrix elements.

$$
\begin{aligned}
A & =\sum_{H_{i}=h, H} \sum_{f=b, t} A_{(1)}\left(f, H_{i}\right)+\sum_{f=b, t} A_{(2)}(f)+\sum_{H_{i}=h, H} \sum_{s=\tilde{t}_{1}, \tilde{t}_{2}} A_{(3+4)}\left(s, H_{i}\right) \\
& +\sum_{s=\tilde{t}_{1}, \tilde{t}_{2}} A_{(5+6)}(s)+\sum_{s^{\prime}=\tilde{t}_{1}, \tilde{t}_{2}} \sum_{s=\tilde{t}_{1}, \tilde{t}_{2}} A_{(7+8)}\left(s, s^{\prime}\right) \\
B & =\sum_{f=b, t} B_{(2)}(f)+\sum_{s^{\prime}=\tilde{t}_{1}, \tilde{t}_{2}} \sum_{s=\tilde{t}_{1}, \tilde{t}_{2}} B_{(8)}\left(s, s^{\prime}\right),
\end{aligned}
$$

The matrix elements $A_{(i)}$ and $B_{(i)}$ read,

$$
A_{(1)}\left(f, H_{i}\right)=\frac{g_{H_{i} h h} g_{H_{i} f f}}{m_{f}} \frac{F_{\triangleright}^{(1 / 2)}\left(m_{f}\right)}{\hat{s}-m_{H_{i}}^{2}+i \Gamma_{H_{i}} m_{H_{i}}}
$$




$$
\begin{aligned}
A_{(2)}(f) & =\left(\frac{g_{h f f}}{m_{f}}\right)^{2} F_{\square}^{(1 / 2)}\left(m_{f}\right) \\
B_{(2)}(f) & =\left(\frac{g_{h f f}}{m_{f}}\right)^{2} G_{\square}^{(1 / 2)}\left(m_{f}\right) \\
A_{(3+4)}\left(s, H_{i}\right) & =\frac{g_{H_{i} h h} g_{H_{i} s s}}{2 m_{s}^{2}} \frac{F_{\triangleright}^{(0)}\left(m_{s}\right)}{\hat{s}-m_{H_{i}}^{2}+i \Gamma_{H_{i}} m_{H_{i}}} \\
A_{(5+6)}(s) & =-\frac{g_{h h s s}}{2 m_{s}^{2}} F_{\triangleright}^{(0)}\left(m_{s}\right) \\
A_{(7+8)}\left(s, s^{\prime}\right) & =\frac{g_{h s s^{\prime}}^{2}}{2 m_{s}^{2} m_{s^{\prime}}^{2}} F_{\square}^{(0)}\left(m_{s}, m_{s^{\prime}}\right) \\
B_{(8)}\left(s, s^{\prime}\right) & =\frac{g_{h s s^{\prime}}^{2}}{2 m_{s}^{2} m_{s^{\prime}}^{2}} G_{\square}^{(0)}\left(m_{s}, m_{s^{\prime}}\right)
\end{aligned}
$$

Expressions for the form factors $\left(F_{\triangleright}, F_{\square}, G_{\square}\right)$ are given in Appendix A. $\hat{s}, \hat{t}, \hat{u}$ are Mandelstam variables. We include the dominant fermion contributions from top and bottom loops, and sfermion contributions from stops and sbottoms. The MSSM couplings $(g)$, $\operatorname{masses}\left(m_{H_{i}}\right)$, and decay widths $\left(\Gamma_{H_{i}}\right)$ of Higgs bosons are calculated by using FeynHiggs2.14.3 [17-23]. We choose $Q=m_{h h} / 2$, where $m_{h h}$ is the invariant mass of the SM-like Higgs pair.

We separate the differential cross sections into resonant, non-resonant, and interference contributions as

$$
\begin{aligned}
\frac{d \hat{\sigma}_{r e s}}{d \hat{t}} & =\frac{\left(\alpha_{s}(Q)\right)^{2}}{2^{13} \pi^{3}}\left|A_{r e s}\right|^{2}, \\
\frac{d \hat{\sigma}_{n r}}{d \hat{t}} & =\frac{\left(\alpha_{s}(Q)\right)^{2}}{2^{13} \pi^{3}}\left(\left|A_{n r}\right|^{2}+\left|B_{n r}\right|^{2}\right), \\
\frac{d \hat{\sigma}_{i n t}}{d \hat{t}} & =\frac{\left(\alpha_{s}(Q)\right)^{2}}{2^{13} \pi^{3}}\left(2 \operatorname{Re}\left(A_{r e s} \cdot A_{n r}^{*}\right)\right),
\end{aligned}
$$

where

$$
\begin{aligned}
A_{r e s} & =\sum_{f=b, t} A_{(1)}(f, H)+\sum_{s=\tilde{t}_{1}, \tilde{t}_{2}} A_{(3+4)}(s, H), \\
A_{n r} & =A-A_{r e s}, \\
B_{n r} & =B .
\end{aligned}
$$

The resonant amplitude is defined such that it has a pole in the region of interest. The non-resonant amplitude includes all other Feynman diagrams, and the interference cross section corresponds to the interference between resonant and non-resonant amplitude, as the name suggests.

The total cross section corresponds to each partonic cross section can be obtained by using

$$
\sigma(p p \rightarrow g g \rightarrow h h)=\int_{\left(2 m_{h}\right)^{2}}^{(14 \mathrm{TeV})^{2}} d \hat{s} \frac{d \mathcal{L}_{g g}}{d \hat{s}} \hat{\sigma}
$$

where $\frac{d \mathcal{L}_{g g}}{d \hat{s}}$ is the differential gluon-gluon luminosity as defined in [24]. 


\section{$2.2 \quad$ NMSSM}

The NMSSM has one extra supermultiplet as compared to the MSSM, i.e. the gauge singlet chiral superfield $\hat{S}$, which contains one complex spin-0 singlet $(S)$ and one spin- $1 / 2$ singlino $(\tilde{S})$. $S$ gives rise to one neutral CP-even Higgs and one neutral CP-odd Higgs. In this article, we only consider the $\mathbb{Z}_{3}$-invariant NMSSM, which has the scale invariant superpotential $W_{\text {Higgs }}$ given by

$$
W_{\mathrm{Higgs}}=\lambda \hat{S} \hat{H}_{u} \cdot \hat{H}_{d}+\frac{\kappa}{3} \hat{S}^{3},
$$

where $W_{\text {Higgs }}$ is the part of superpotential that depends exclusively on Higgs superfields $\hat{H}_{u}, \hat{H}_{d}$, and $\hat{S}$. In the next paragraph, we will briefly introduce the notations and sign conventions of relevant NMSSM parameters that appear in this paper. Please refer to the review paper [25] for more details about the NMSSM.

The first term in Eq. (2.13) replaces the $\mu \hat{H}_{u} \cdot \hat{H}_{d}$ term in MSSM superpotential, and generates the effective $\mu$-term at electroweak scale when $S$ acquires a non-vanishing vev:

$$
\mu_{\mathrm{eff}}=\lambda\langle S\rangle
$$

$v_{u}$ and $v_{d}$ are vevs of $H_{u}^{0}$ and $H_{d}^{0}$ respectively, and

$$
\tan \beta=\frac{v_{u}}{v_{d}} .
$$

$A_{\lambda}$ and $A_{\kappa}$ are trilinear soft SUSY breaking couplings correspond to the first and second term in eq.(2.13) respectively. $M_{1}, M_{2}$, and $M_{3}$ are soft SUSY breaking masses of $U(1)_{Y}$ gaugino, $S U(2)$ gauginos, and $S U(3)$ gauginos respectively. We choose $\tan \beta$ and $\lambda$ to be positive, while $\mu_{\text {eff }}, \kappa, A_{\lambda}, A_{\kappa}$ can have both signs.

The set of leading order Feynman diagrams for $g g \rightarrow h h$ in NMSSM is the same as the MSSM case, except that there are three neutral CP-even Higgs bosons $\left(H_{i}=H_{1}, H_{2}, H_{3}\right.$, ordered by ascending mass) in NMSSM. Thus, Eq. (2.2-2.8) are still valid in NMSSM case. The NMSSM couplings, masses, and decay rates of Higgs bosons are calculated by using NMSSMTools-5.4.1 [26-31].

\section{Phenomenological Study}

\subsection{MSSM}

In the MSSM study, for the stops to contribute in a relevant way, the stop should not be too far away from the weak scale [13]. Therefore, we chose the lighter stop mass to be $600 \mathrm{GeV}$, which is right above the current LHC limit [32,33]. To allow for large mixings in the stop sector, which can enhance the di-Higgs production rate through diagrams (7) and (8) in Fig. 1, we choose the heavier stop mass to be $5 \mathrm{TeV}$. The stop mixing parameter $X_{t}$ is chosen so that the SM-like Higgs mass is $125 \mathrm{GeV}$. We calculated the SM-like Higgs mass using FeynHiggs-2.14.3 [17-23], and found that in the region of interest, there 
always exists a value of $X_{t}$ such that $m_{h}=125 \mathrm{GeV}$. Futhermore, $X_{t}$ cannot be too large in order to have a stable SM-like vacuum. Here, we use the approximate bound [34].

$$
\left(X_{t}+\frac{\mu}{\tan \beta}\right)^{2} \leq\left(3.4\left(m_{\tilde{Q}_{3}}^{2}+m_{\tilde{t}_{R}}^{2}\right)+0.5\left|m_{\tilde{Q}_{3}}^{2}-m_{\tilde{t}_{R}}^{2}\right|\right)+60\left(\frac{m_{Z}^{2}}{2} \cos 2 \beta+m_{A}^{2} \cos ^{2} \beta\right) .
$$

We checked that this bound is satisfied by every point in the parameter space that is shown in Fig.2.

Then we fix the masses and the mixings in the sbottom sector by imposing $m_{\tilde{t}_{R}}=m_{\tilde{b}_{R}}$, and $A_{t}=A_{b}$. We ignore the contributions from all other sfermions and electroweakinos. When the electroweakinos are lighter than $m_{H} / 2$, the heavy Higgs starts to decay to a pair of electroweakinos, which reduces its branching ratio to a pair of SM Higgs, and therefore reduces the resonant di-Higgs production cross section. As we aim to understand how large the di-Higgs production cross section can be at the LHC, we ignore the contributions from electroweakinos.

Based on those considerations, we keep $m_{A}$, the mass of the CP-odd Higgs boson, and $\tan \beta$, the ratio between two vevs, as free parameters in our study. The ranges of $m_{A}$ and $\tan \beta$ are restricted by several experimental observations and theoretical requirements as shown in Fig.2. First, the Higgs couplings depend strongly on the mixing between the SM-like Higgs and the new CP-even Higgs, and in the region of interest, can deviate significantly from the SM. Therefore, the parameter space is restricted by precision Higgs measurements, and the most stringent limit comes from the Higgs boson coupling to bottom quark measurement at ATLAS [6]. The blue shaded region with the thin solid boundary line is excluded by the precision measurement of Higgs couplings at $\sqrt{s}=13 \mathrm{TeV}[5,6]$. Second, the heavy Higgs couples to the SM particles, and can be searched for through its decays. The most sensitive channel is in the di-tau final state, and the blue shaded region with the thick solid boundary line is excluded by the search for the additional neutral Higgs bosons in the di-tau final state at $\sqrt{s}=13 \mathrm{TeV}[35,36]$.

We calculate the leading order di-Higgs production cross section as described in the previous section, and the results are shown in Fig. 3. To account for the modifications in the Higgs decay, we show the results in the two most sensitive channels, $b b \gamma \gamma$ and $b b \tau \tau$, according to the ATLAS projections [37]. In the region of interest, $B(h \rightarrow b \bar{b}) B\left(h \rightarrow \tau^{+} \tau^{-}\right)$ is always enhanced, and it can be enhanced up to $25 \%$ compared to the SM value, while $B(h \rightarrow b \bar{b}) B(h \rightarrow \gamma \gamma)$ is suppressed in most of the parameter space, and it can be suppressed up to $14 \%$ compared to the SM value. The di-Higgs production rate can be enhanced significantly in the region where $m_{A}$ and $\tan \beta$ are small. For instance, when $m_{A}=400 \mathrm{GeV}$ and $\tan \beta=2, \sigma\left(p p \rightarrow h h \rightarrow b \bar{b} \tau^{+} \tau^{-}\right) / \mathrm{SM}=13.7$ and $\sigma(p p \rightarrow h h \rightarrow$ $b \bar{b} \gamma \gamma) / \mathrm{SM}=11.3$. This enhancement is mainly due to the large resonant contribution of di-Higgs production in this region, which can also be seen in Fig.4. In Fig. 4, we plot the resonant production of di-Higgs normalized to the SM production rate. The production rate can be a few of the SM value when $m_{A}$ and $\tan \beta$ are small, and decreases quickly as $m_{A}$ and $\tan \beta$ increase as expected.

Outside the region where di-Higgs production receives a large correction from the resonant production, the modifications come from the non-resonant production. The non- 


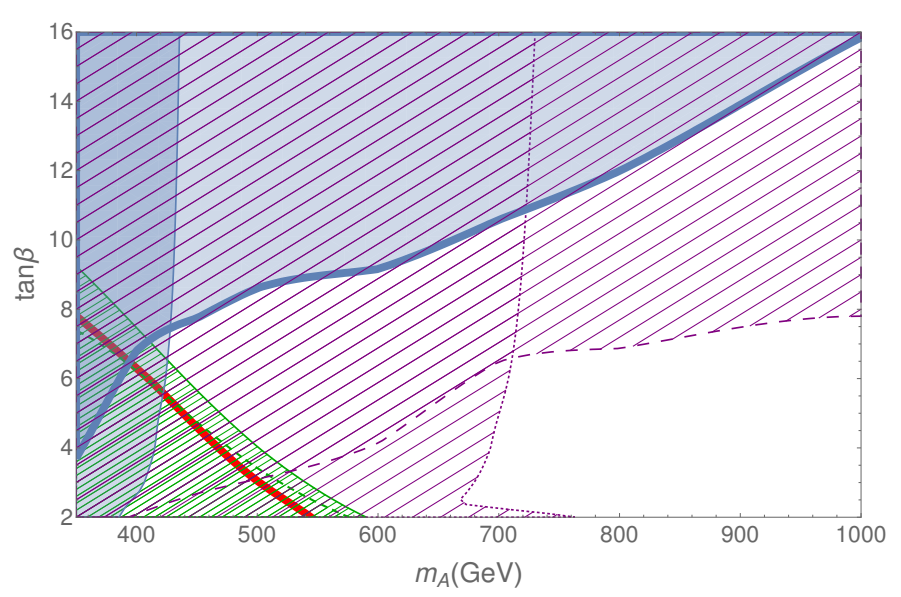

Figure 2. The MSSM parameter space with current experimental constraints and projected sensitivities. The blue shaded region is excluded by current experiment constraints. The purple and green shaded region can be reached by HL-LHC. The red solid line represents the case when resonant cross section equals to non-resonant cross section.
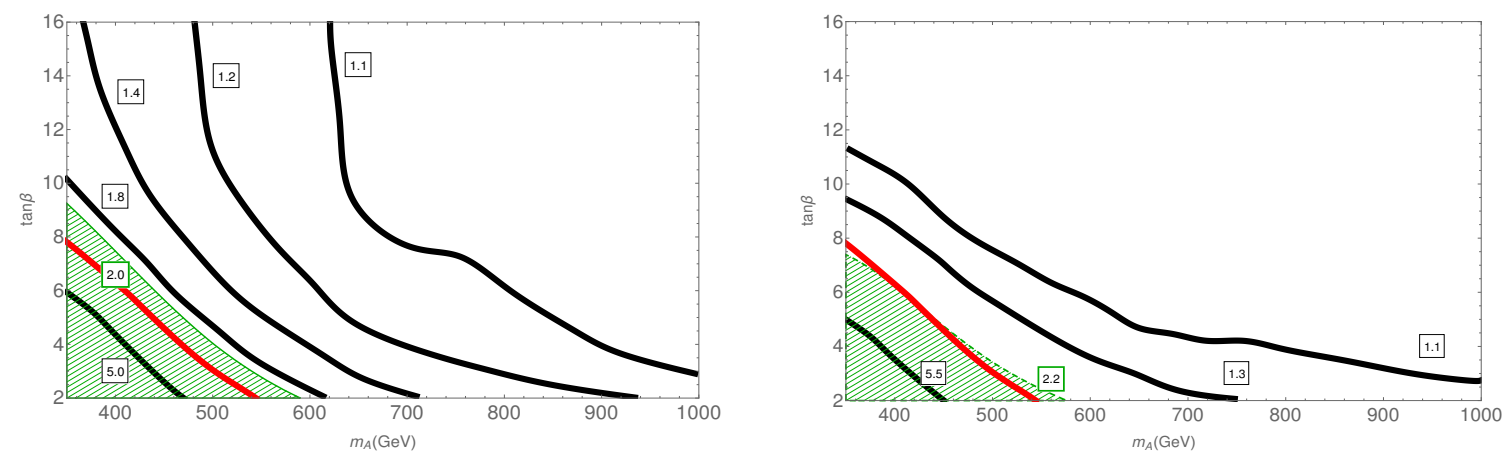

Figure 3. Contour plots for $\sigma\left(p p \rightarrow h h \rightarrow b \bar{b} \tau^{+} \tau^{-}\right)$(left panel) and $\sigma(p p \rightarrow h h \rightarrow b \bar{b} \gamma \gamma)$ (right panel) normalized to SM value at $\sqrt{s}=14 \mathrm{TeV}$. The cross sections in the shaded regions are outside the $95 \%$ CL of the projected di-Higgs production cross section at HL-LHC.

resonant di-Higgs production is very close to the SM contribution in the whole region of interest. Our calculations show that $\kappa_{t}=g_{h t t} / g_{h t t}^{\mathrm{SM}}$ varies between 0.95 and 1 , while $\lambda_{3}=\left(g_{h h h}-g_{h h h}^{\mathrm{SM}}\right) / g_{h h h}^{\mathrm{SM}}$ varies between -0.24 and -0.12 in the parameter space of interest. The decrease in the SM-like Higgs self-coupling reduces the destructive interference between the triangle diagram and the fermionic box diagram (i.e. first and second diagram in Fig.1), hence it enhances non-resonant di-Higgs production cross section. However, this effect is offset by a small decrease in $\kappa_{t}$. The box diagram, which dominates over the triangle diagram, is proportional to $\kappa_{t}^{4}$, so the di-Higgs rate is very sensitive to the value of $\kappa_{t}$. Therefore, a small decrease in $\kappa_{t}$ offsets the decrease in $\lambda_{3}$. As a result, the non-resonant di-Higgs cross section is only larger than the SM di-Higgs production cross section by a few percent in most of the parameter space. The interference between the resonant and non-resonant amplitude is small over the whole parameter space, which we plot in Fig.5. Contribution from stop loops is less than $3 \%$ of the total di-Higgs production cross section. 


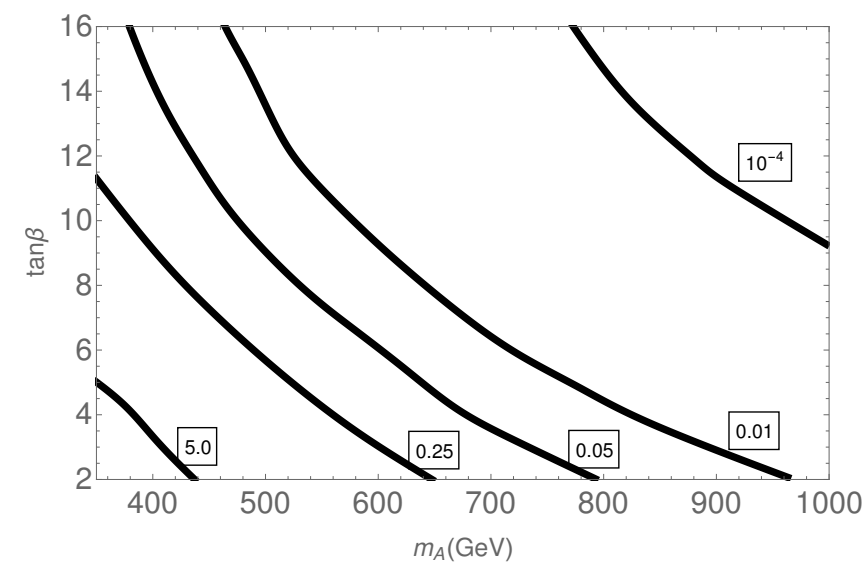

Figure 4. Resonant contribution of di-Higgs production cross section (normalized to SM value) at $\sqrt{s}=14 \mathrm{TeV}$.

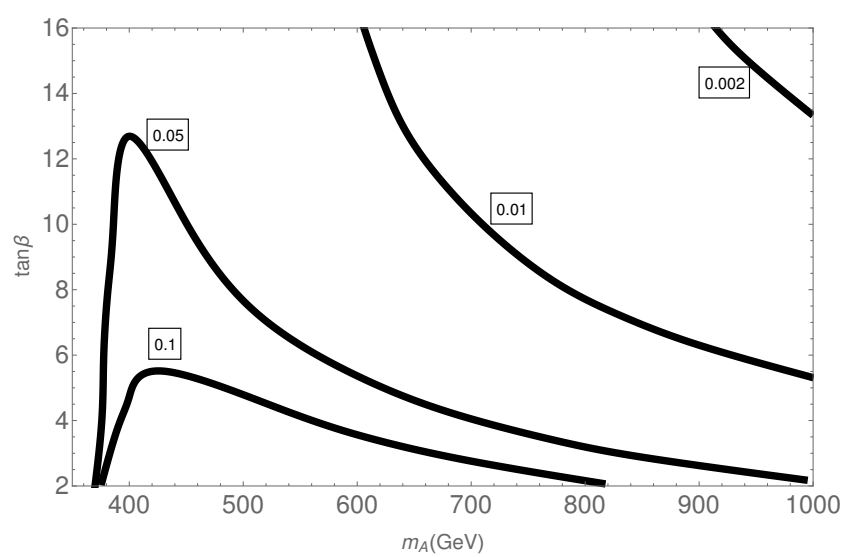

Figure 5. Interference (normalized to SM value) between resonant and non-resonant amplitude at $\sqrt{s}=14 \mathrm{TeV}$.

Therefore, in the MSSM case, the major modification to di-Higgs production is from the new resonance, which is the new CP-even neutral Higgs, and the modifications to the branching ratios.

We also study the complementarity between di-Higgs and other Higgs studies. In Fig. 2, we compare the projected sensitivity in the $b b \gamma \gamma$ and the $b b \tau \tau$ channels with other searches. We separate the parameter space into two regions, the one below the red solid line is dominated by resonant production, while the region above the red solid line is dominated by non-resonant production. The green shaded region with solid boundary line and the dashed boundary line show the region that can be excluded by the non-resonant $b b \tau \tau$ and $b b \gamma \gamma$ searches at HL-LHC with $3000 \mathrm{fb}^{-1}$ respectively. The projected sensitivity for the resonant production is not available at present, but we do expect that is better compared to the sensitivity for the non-resonant production. Therefore, the whole green shaded region can be probed by HL-LHC. The purple shaded region with the dotted boundary line shows the region can be tested by the projected Higgs boson couplings measurements at HL-LHC 


\begin{tabular}{|c|ccc|ccccccccccc|}
\hline & $\tan \beta$ & $\lambda$ & $\kappa$ & $A_{\lambda}$ & $A_{\kappa}$ & $\mu_{\text {eff }}$ & $M_{1}$ & $\begin{array}{c}M_{2} \\
\left(\mathrm{M} / M_{3}\right.\end{array}$ & $A_{t}$ & $A_{b}$ & $A_{\tau}$ & $m_{\tilde{Q}_{3}}$ & $m_{\tilde{L}_{3}}$ \\
\hline $\min$ & 1 & 0 & -0.7 & -1 & -1 & -0.5 & 0.1 & 0.2 & 1.3 & -6 & -6 & -3 & 0.6 & 0.6 \\
\hline $\max$ & 10 & 0.7 & 0.7 & 1 & 1 & 0.5 & 1 & 2 & 7 & 6 & 6 & 3 & 4 & 4 \\
\hline
\end{tabular}

Table 1. NMSSM parameter space.

[38]. The purple shaded region with the dashed boundary line shows the projected reach for the additional neutral Higgs bosons in the di-tau final state at HL-LHC at 95\% C.L. [37]. From Fig.2, a large fraction of the parameter space that is not excluded by current experimental constraints will be tested by the HL-LHC experiments (i.e. the purple and green region). The di-Higgs production is not as sensitive as other searches, but provides a complementary probe at the HL-LHC.

\subsection{NMSSM}

In the NMSSM study, we perform a random scan over the NMSSM parameter space using NMSSMTools-5.4.1 as there are more relevant parameters. A similar study can be found in [39]. The range of the parameters is as shown in Tab.1. We also assume

$$
m_{\tilde{t}_{R}}=m_{\tilde{Q}_{3}}, \quad m_{\tilde{\tau}_{R}}=m_{\tilde{L}_{3}}, \quad m_{\tilde{b}_{R}}=3 \mathrm{TeV},
$$

although we expect the sfermion contributions are negligible, as in the MSSM case. We require the mass of one of the NMSSM neutral CP-even Higgs bosons to be $125.26 \pm 3 \mathrm{GeV}$ to accommodate experimental and theoretical uncertainties up to $3 \mathrm{GeV}$. Besides that, $\lambda$ and $\kappa$ have to be sufficiently small so that perturbation theory remains valid. Here, we use [40]

$$
\lambda^{2}+\kappa^{2}<0.7^{2}
$$

Constraints from collider experiments (LEP, Tevatron, LHC) and dark matter direct detection experiments are also checked by using NMSSMTools-5.4.1 [26-31, 41-43]. All points in Fig. 6 also satisfy the constraint on dark matter relic density from Planck measurement [44] (including $+10 \%$ uncertainty in theoretical calculation)

$$
\Omega_{\tilde{\chi}_{1}^{0}} h^{2} \leq 0.131
$$

Our results for the NMSSM are shown in Fig.6, for each points surviving all constraints, we plot the di-Higgs production cross section in the $b b \tau^{+} \tau^{-}$channel (upper panel) and the $b b \gamma \gamma$ channel (lower panel). In the region of interest, $B(h \rightarrow b \bar{b}) B\left(h \rightarrow \tau^{+} \tau^{-}\right)$can be enhanced up to $16 \%$ compared to the SM value, and $B(h \rightarrow b \bar{b}) B(h \rightarrow \gamma \gamma)$ can be suppressed up to $10 \%$ compared to the SM value. The NMSSM di-Higgs production cross sections can be enhanced significantly compared to the SM. Similar to the MSSM case, the interference between the resonant and non-resonant amplitude and the contributions from squark loops are small over the whole parameter space. When the new CP-even Higgs states are light, the dominant contribution is from resonant di-Higgs production. The resonant di-Higgs rate can be a few times the SM rate. The non-resonant contribution of 

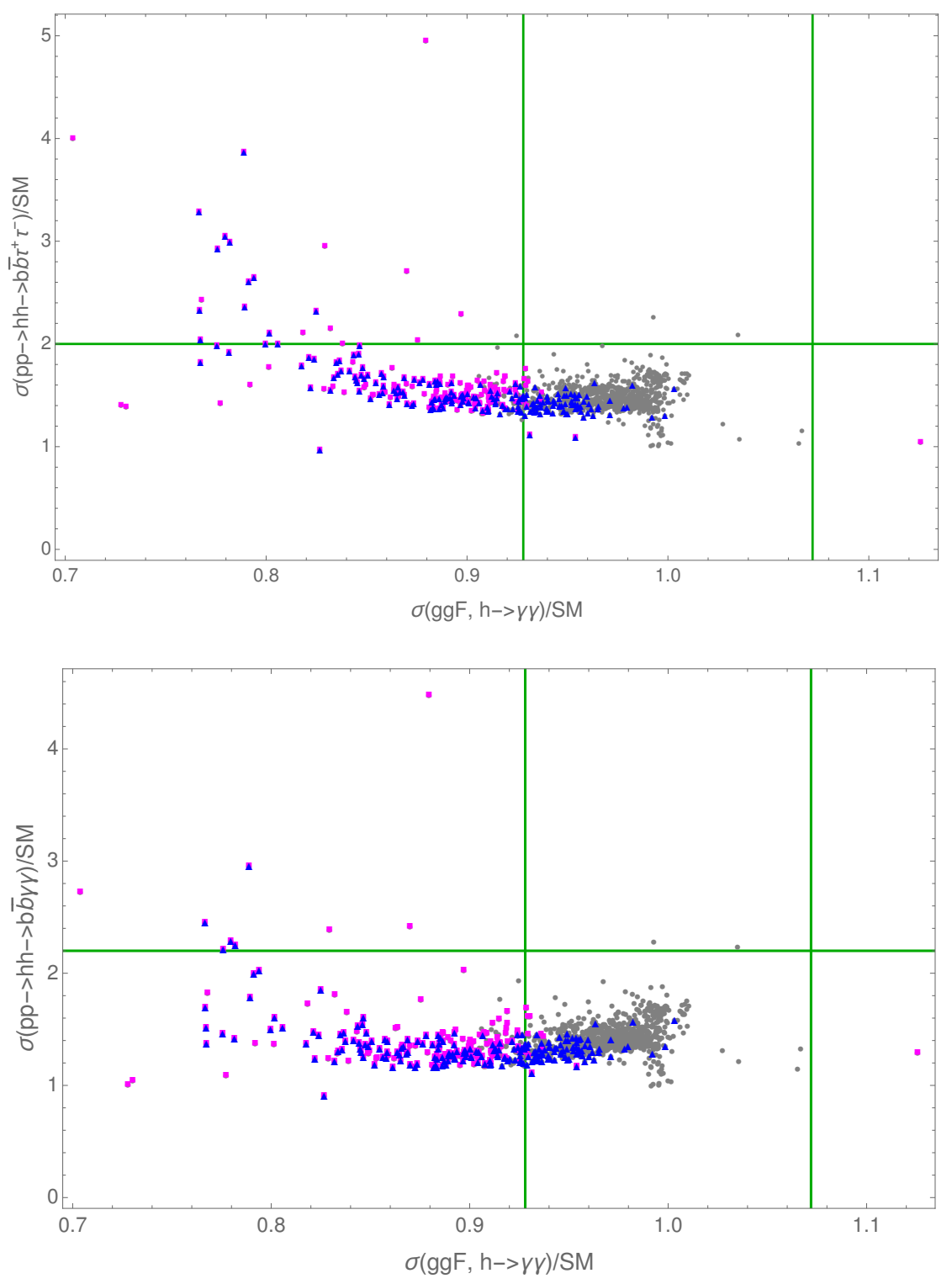

Figure 6. Scatter plots for $\sigma\left(p p \rightarrow h h \rightarrow b \bar{b} \tau^{+} \tau^{-}\right)$(upper panel) and $\sigma(p p \rightarrow h h \rightarrow b \bar{b} \gamma \gamma)$ (lower panel) normalized to SM value as a function of the SM-like Higgs boson production cross section via gluon-gluon fusion, with the SM-like Higgs boson decaying into $\gamma \gamma$ pair. All points satisfy current experimental constraints. The horizontal line is the 95\% CL upper limit of the projected di-Higgs production cross section at HL-LHC. The vertical lines are the 95\% CL limits of the projected $\sigma(g g F, h \rightarrow \gamma \gamma)$ at HL-LHC. The magenta dots are outside the $95 \%$ CL of the projected SM-like Higgs boson production cross sections via other production modes and decay modes at HL-LHC. The blue dots are outside the 95\% CL of the projected search for the additional neutral Higgs Bosons in the di-tau final state at HL-LHC.

di-Higgs production is generally enhanced by $20 \%$ to $60 \%$, which comes from the decrease in the SM-like Higgs self-coupling. In the region of interest, $\delta_{3}$ generally varies between -0.3 and -0.58 , resulting in the $20 \%$ to $60 \%$ enhancement in the non-resonant production. Here we use the projected sensitivity of the non-resonant production, and we expect it to 
be better for resonant productions. Unlike the MSSM case, $\kappa_{t}$ is very close to unity in the NMSSM case, and therefore, the enhancement due to the suppression in $\lambda_{3}$ is not offset by the suppression in $\kappa_{t}$. In most of the parameter space, the di-Higgs rate is enhanced through the suppression in $\lambda_{3}$.

The horizontal lines in Fig. 6 show the projected sensitivity at the HL-LHC for the $b b \tau \tau$ channel (upper panel), and the $b b \gamma \gamma$ channel (lower panel). Most points lie below the horizontal lines, meaning the HL-LHC di-Higgs production measurements have very limited sensitivity in most regions of interest.

We also study the complementarity between di-Higgs and other Higgs measurements. In terms of precision Higgs measurements, we found that $h \rightarrow \gamma \gamma$ from gluon fusion is the most sensitive channel among all Higgs boson production modes and decay channels at the HL-LHC. To compare with precision Higgs measurements, we plot the $h \rightarrow \gamma \gamma$ cross section from gluon fusion and overlay the projected sensitivity in this channel as the vertical lines at the HL-LHC. The $h \rightarrow \gamma \gamma$ measurement can probe a large fraction of the parameter space, and almost all points that can be tested by the di-Higgs measurement, can be tested by the $h \rightarrow \gamma \gamma$ measurement. To consider the sensitivity in precision Higgs measurements, we use magenta dots to represent points that can be tested by other Higgs coupling measurements. Most points that can be tested by the $h \rightarrow \gamma \gamma$ measurement can be probed by other Higgs measurements as well, such as $h \rightarrow Z Z$ and $h \rightarrow W W$ measurements.

For direct searches of the heavy Higgs, we found that the search for heavy neutral Higgs bosons in the di-tau final state at HL-LHC [37] is a good complementary probe. We use blue dots to represent the points that can be tested in the heavy Higgs to di-tau channel. From Fig.6, we can see that many blue dots lie between the two vertical lines, and below the horizontal line, showing direct searches of a heavy Higgs provides a complementary probe to precision Higgs and di-Higgs measurements. Combining di-Higgs, precision Higgs, and direct searches of the heavy Higgs, a large set of parameter points can be tested at the HL-LHC.

\section{Conclusions}

In this work, we have calculated the di-Higgs production cross section in the MSSM and the NMSSM at the one loop level. We include the possible resonant contribution from new CP-even neutral Higgs states, new colored particles, and possible modifications in the Higgs couplings. We found that in both cases, the di-Higgs production can be enhanced significantly through a new resonance. As the new CP-even neutral Higgs bosons become heavy, di-Higgs production only enhances moderately due to the suppression in the Higgs self-couplings. The di-Higgs rate in the $b b \tau^{+} \tau^{-}$final state can be further enhanced through the enhanced branching ratios. Given the current stop limit, we found the contributions from stops and other sfermions are small. Also, the interference effect is found to be small.

We further study the complementarity of di-Higgs measurement to other Higgs studies, including the precision measurement of the Higgs couplings and the search for new Higgs bosons. We found that there is a strong correlation in di-Higgs production rate and single 
Higgs production rate, especially outside the region where resonant di-Higgs dominates. Due to the complexity of the signature, and the small production cross section, di-Higgs measurements are not as sensitive as single Higgs measurements. The direct searches to new Higgs bosons, on the other hand, provide a complementary probe.

\section{Acknowledgments}

We thank I. Lewis, K. Hagowara, and S. Heinemeyer for helpful discussions. This work is supported by University of Nebraska-Lincoln, National Science Foundation under grant number PHY-1820891, and the NSF Nebraska EPSCoR under grant number OIA-1557417. 


\section{A Form Factors}

For the partonic process $g\left(p_{1}\right) g\left(p_{2}\right) \rightarrow h\left(k_{1}\right) h\left(k_{2}\right)$,

$$
\begin{aligned}
& F_{\triangleright}^{(1 / 2)}\left(m_{f}\right)=\frac{2 m_{f}^{2}}{\hat{s}}\left[2+\left(4 m_{f}^{2}-\hat{s}\right) C_{0}\left(0,0, \hat{s}, m_{f}^{2}, m_{f}^{2}, m_{f}^{2}\right)\right], \\
& F_{\square}^{(1 / 2)}\left(m_{f}\right)=\frac{2 m_{f}^{2}}{\hat{s}}\left\{m_{f}^{2}\left(8 m_{f}^{2}-\hat{s}-2 m_{h}^{2}\right)\right. \\
& {\left[D_{0}\left(0,0, m_{h}^{2}, m_{h}^{2}, \hat{s}, \hat{u}, m_{f}^{2}, m_{f}^{2}, m_{f}^{2}, m_{f}^{2}\right)+D_{0}\left(0,0, m_{h}^{2}, m_{h}^{2}, \hat{s}, \hat{t}, m_{f}^{2}, m_{f}^{2}, m_{f}^{2}, m_{f}^{2}\right)\right.} \\
& \left.+D_{0}\left(0, m_{h}^{2}, 0, m_{h}^{2}, \hat{t}, \hat{u}, m_{f}^{2}, m_{f}^{2}, m_{f}^{2}, m_{f}^{2}\right)\right] \\
& +\frac{\hat{u} \hat{t}-m_{h}^{4}}{\hat{s}}\left(4 m_{f}^{2}-m_{h}^{2}\right) D_{0}\left(0, m_{h}^{2}, 0, m_{h}^{2}, \hat{t}, \hat{u}, m_{f}^{2}, m_{f}^{2}, m_{f}^{2}, m_{f}^{2}\right) \\
& +2+4 m_{f}^{2} C_{0}\left(0,0, \hat{s}, m_{f}^{2}, m_{f}^{2}, m_{f}^{2}\right) \\
& +\frac{2}{\hat{s}}\left(m_{h}^{2}-4 m_{f}^{2}\right)\left[\left(\hat{t}-m_{h}^{2}\right) C_{0}\left(0, m_{h}^{2}, \hat{t}, m_{f}^{2}, m_{f}^{2}, m_{f}^{2}\right)\right. \\
& \left.\left.+\left(\hat{u}-m_{h}^{2}\right) C_{0}\left(0, m_{h}^{2}, \hat{u}, m_{f}^{2}, m_{f}^{2}, m_{f}^{2}\right)\right]\right\}, \\
& G_{\square}^{(1 / 2)}\left(m_{f}\right)=\frac{m_{f}^{2}}{\hat{s}}\left\{2\left(8 m_{f}^{2}+\hat{s}-2 m_{h}^{2}\right)\right. \\
& \left\{m _ { f } ^ { 2 } \left[D_{0}\left(0,0, m_{h}^{2}, m_{h}^{2}, \hat{s}, \hat{u}, m_{f}^{2}, m_{f}^{2}, m_{f}^{2}, m_{f}^{2}\right)+D_{0}\left(0,0, m_{h}^{2}, m_{h}^{2}, \hat{s}, \hat{t}, m_{f}^{2}, m_{f}^{2}, m_{f}^{2}, m_{f}^{2}\right)\right.\right. \\
& \left.\left.+D_{0}\left(0, m_{h}^{2}, 0, m_{h}^{2}, \hat{t}, \hat{u}, m_{f}^{2}, m_{f}^{2}, m_{f}^{2}, m_{f}^{2}\right)\right]-C_{0}\left(m_{h}^{2}, m_{h}^{2}, \hat{s}, m_{f}^{2}, m_{f}^{2}, m_{f}^{2}\right)\right\} \\
& -2\left\{\hat{s} C_{0}\left(0,0, \hat{s}, m_{f}^{2}, m_{f}^{2}, m_{f}^{2}\right)+\left(\hat{t}-m_{h}^{2}\right) C_{0}\left(0, m_{h}^{2}, \hat{t}, m_{f}^{2}, m_{f}^{2}, m_{f}^{2}\right)\right. \\
& \left.+\left(\hat{u}-m_{h}^{2}\right) C_{0}\left(0, m_{h}^{2}, \hat{u}, m_{f}^{2}, m_{f}^{2}, m_{f}^{2}\right)\right\} \\
& +\frac{1}{u t-m h^{4}}\left\{\hat{s} \hat{u}\left(8 \hat{u} m_{f}^{2}-u^{2}-m_{h}^{4}\right) D_{0}\left(0,0, m_{h}^{2}, m_{h}^{2}, \hat{s}, \hat{u}, m_{f}^{2}, m_{f}^{2}, m_{f}^{2}, m_{f}^{2}\right)\right. \\
& +\hat{s} \hat{t}\left(8 \hat{t} m_{f}^{2}-\hat{t}^{2}-m_{h}^{4}\right) D_{0}\left(0,0, m_{h}^{2}, m_{h}^{2}, \hat{s}, \hat{t}, m_{f}^{2}, m_{f}^{2}, m_{f}^{2}, m_{f}^{2}\right) \\
& +\left(8 m_{f}^{2}+\hat{s}-2 m_{h}^{2}\right)\left\{\hat{s}\left(\hat{s}-2 m_{h}^{2}\right) C_{0}\left(0,0, \hat{s}, m_{f}^{2}, m_{f}^{2}, m_{f}^{2}\right)\right. \\
& +\hat{s}\left(\hat{s}-4 m_{h}^{2}\right) C_{0}\left(m_{h}^{2}, m_{h}^{2}, \hat{s}, m_{f}^{2}, m_{f}^{2}, m_{f}^{2}\right) \\
& +2 \hat{t}\left(m_{h}^{2}-\hat{t}\right) C_{0}\left(0, m_{h}^{2}, \hat{t}, m_{f}^{2}, m_{f}^{2}, m_{f}^{2}\right) \\
& \left.\left.\left.+2 \hat{u}\left(m_{h}^{2}-\hat{u}\right) C_{0}\left(0, m_{h}^{2}, \hat{u}, m_{f}^{2}, m_{f}^{2}, m_{f}^{2}\right)\right\}\right\}\right\} \\
& F_{\triangleright}^{(0)}\left(m_{s}\right)=\frac{-2 m_{s}^{2}}{\hat{s}}\left(1+2 m_{s}^{2} C_{0}\left(0,0, \hat{s}, m_{f}^{2}, m_{f}^{2}, m_{f}^{2}\right)\right) \\
& F_{\square}^{(0)}\left(m_{s}, m_{s^{\prime}}\right)=\frac{-4 m_{s}^{2} m_{s^{\prime}}^{2}}{\hat{s}}\left\{\frac{m_{h}^{2}-\hat{t}}{2 \hat{s}}\left[C_{0}\left(m_{h}^{2}, 0, \hat{t}, m_{s^{\prime}}^{2}, m_{s}^{2}, m_{s}^{2}\right)+C_{0}\left(m_{h}^{2}, 0, \hat{t}, m_{s}^{2}, m_{s^{\prime}}^{2}, m_{s^{\prime}}^{2}\right)\right]\right. \\
& +\frac{m_{h}^{2}-\hat{u}}{2 \hat{s}}\left[C_{0}\left(m_{h}^{2}, 0, \hat{u}, m_{s^{\prime}}^{2}, m_{s}^{2}, m_{s}^{2}\right)+C_{0}\left(m_{h}^{2}, 0, \hat{u}, m_{s}^{2}, m_{s^{\prime}}^{2}, m_{s^{\prime}}^{2}\right)\right] \\
& +\frac{1}{2}\left(m_{s^{\prime}}^{2}-m_{s}^{2}+\frac{\hat{u} \hat{t}-m_{h}^{4}}{\hat{s}}\right) D_{0}\left(m_{h}^{2}, 0, m_{h}^{2}, 0, \hat{t}, \hat{u}, m_{s^{\prime}}^{2}, m_{s}^{2}, m_{s}^{2}, m_{s^{\prime}}^{2}\right) \\
& +m_{s}^{2}\left[D_{0}\left(m_{h}^{2}, 0, m_{h}^{2}, 0, \hat{t}, \hat{u}, m_{s^{\prime}}^{2}, m_{s}^{2}, m_{s}^{2}, m_{s^{\prime}}^{2}\right)+D_{0}\left(m_{h}^{2}, m_{h}^{2}, 0,0, \hat{s}, \hat{t}, m_{s}^{2}, m_{s^{\prime}}^{2}, m_{s}^{2}, m_{s}^{2}\right)\right. \\
& \left.\left.+D_{0}\left(m_{h}^{2}, m_{h}^{2}, 0,0, \hat{s}, \hat{u}, m_{s}^{2}, m_{s^{\prime}}^{2}, m_{s}^{2}, m_{s}^{2}\right)\right]\right\}
\end{aligned}
$$




$$
\begin{aligned}
G_{\square}^{(0)}\left(m_{s}, m_{s^{\prime}}\right) & =\frac{-2 m_{s}^{2} m_{s^{\prime}}^{2}}{\hat{s}\left(m_{h}^{4}-\hat{u} \hat{t}\right)}\left\{\hat{s}\left(2 m_{s}^{2}-2 m_{s^{\prime}}^{2}+\hat{t}+\hat{u}\right) C_{0}\left(0,0, s, m_{s}^{2}, m_{s}^{2}, m_{s}^{2}\right)\right. \\
& -2 \hat{t}\left(m_{h}^{2}-\hat{t}\right) C_{0}\left(m_{h}^{2}, 0, \hat{t}, m_{s^{\prime}}^{2}, m_{s}^{2}, m_{s}^{2}\right)-2 \hat{u}\left(m_{h}^{2}-\hat{u}\right) C_{0}\left(m_{h}^{2}, 0, \hat{u}, m_{s^{\prime}}^{2}, m_{s}^{2}, m_{s}^{2}\right) \\
& -\left(m_{h}^{2}-\hat{t}\right)\left(m_{s}^{2}-m_{s^{\prime}}^{2}\right)\left[C_{0}\left(m_{h}^{2}, 0, \hat{t}, m_{s}^{2}, m_{s^{\prime}}^{2}, m_{s^{\prime}}^{2}\right)+C_{0}\left(m_{h}^{2}, 0, \hat{t}, m_{s^{\prime}}^{2}, m_{s}^{2}, m_{s}^{2}\right)\right] \\
& -\left(m_{h}^{2}-\hat{u}\right)\left(m_{s}^{2}-m_{s^{\prime}}^{2}\right)\left[C_{0}\left(m_{h}^{2}, 0, \hat{u}, m_{s}^{2}, m_{s^{\prime}}^{2}, m_{s^{\prime}}^{2}\right)+C_{0}\left(m_{h}^{2}, 0, \hat{u}, m_{s^{\prime}}^{2}, m_{s}^{2}, m_{s}^{2}\right)\right] \\
& +\left(2 m_{h}^{4}-\hat{t}^{2}-\hat{u}^{2}\right) C_{0}\left(m_{h}^{2}, m_{h}^{2}, \hat{s}, m_{s}^{2}, m_{s^{\prime}}^{2}, m_{s}^{2}\right) \\
& +\left[-\hat{s}\left(m_{s}^{2}-m_{s^{\prime}}^{2}\right)^{2}+\left(m_{s}^{2}+m_{s^{\prime}}^{2}\right)\left(m_{h}^{4}-\hat{u} \hat{t}\right)\right]\left[D_{0}\left(m_{h}^{2}, 0, m_{h}^{2}, 0, \hat{t}, \hat{u}, m_{s^{\prime}}^{2}, m_{s}^{2}, m_{s}^{2}, m_{s^{\prime}}^{2}\right)\right. \\
& \left.+D_{0}\left(m_{h}^{2}, m_{h}^{2}, 0,0, \hat{s}, \hat{t}, m_{s}^{2}, m_{s^{\prime}}^{2}, m_{s}^{2}, m_{s}^{2}\right)+D_{0}\left(m_{h}^{2}, m_{h}^{2}, 0,0, \hat{s}, \hat{u}, m_{s}^{2}, m_{s^{\prime}}^{2}, m_{s}^{2}, m_{s}^{2}\right)\right] \\
& -\left[\hat{s} \hat{t}^{2}+\left(m_{s}^{2}-m_{s^{\prime}}^{2}\right)\left(2 \hat{s} \hat{t}+\hat{u} \hat{t}-m_{h}^{4}\right)\right] D_{0}\left(m_{h}^{2}, m_{h}^{2}, 0,0, \hat{s}, \hat{t}, m_{s}^{2}, m_{s^{\prime}}^{2}, m_{s}^{2}, m_{s}^{2}\right) \\
& \left.-\left[\hat{s} \hat{u}^{2}+\left(m_{s}^{2}-m_{s^{\prime}}^{2}\right)\left(2 \hat{s} \hat{u}+\hat{u} \hat{t}-m_{h}^{4}\right)\right] D_{0}\left(m_{h}^{2}, m_{h}^{2}, 0,0, \hat{s}, \hat{u}, m_{s}^{2}, m_{s^{\prime}}^{2}, m_{s}^{2}, m_{s}^{2}\right)\right\}
\end{aligned}
$$

where

$$
\begin{aligned}
& \hat{s}=\left(p_{1}+p_{2}\right)^{2}, \\
& \hat{t}=\left(p_{1}-k_{1}\right)^{2}, \\
& \hat{u}=\left(p_{1}-k_{2}\right)^{2},
\end{aligned}
$$

and $C_{0}$ and $D_{0}$ are scalar three-point and four-point functions of one-loop integrals, defined as

$$
\begin{aligned}
& C_{0}\left(p_{1}^{2}, p_{2}^{2},\left(p_{1}+p_{2}\right)^{2}, m_{1}^{2}, m_{2}^{2}, m_{3}^{2}\right) \\
& =\frac{\mu^{4-D}}{i \pi^{D / 2} r_{\Gamma}} \int \frac{d^{D} q}{\left[q^{2}-m_{1}^{2}\right]\left[\left(q+p_{1}\right)^{2}-m_{2}^{2}\right]\left[\left(q+p_{1}+p_{2}\right)^{2}-m_{3}^{2}\right]}, \\
& D_{0}\left(p_{1}^{2}, p_{2}^{2}, p_{3}^{2}, p_{4}^{2},\left(p_{1}+p_{2}\right)^{2},\left(p_{2}+p_{3}\right)^{2}, m_{1}^{2}, m_{2}^{2}, m_{3}^{2}, m_{4}^{2}\right) \\
& =\frac{\mu^{4-D}}{i \pi^{D / 2} r_{\Gamma}} \int \frac{d^{D} q}{\left[q^{2}-m_{1}^{2}\right]\left[\left(q+p_{1}\right)^{2}-m_{2}^{2}\right]\left[\left(q+p_{1}+p_{2}\right)^{2}-m_{3}^{2}\right]\left[\left(q+p_{1}+p_{2}+p_{3}\right)^{2}-m_{4}^{2}\right]},
\end{aligned}
$$

where

$$
r_{\Gamma}=\frac{\Gamma^{2}(1-\epsilon) \Gamma(1+\epsilon)}{\Gamma(1-2 \epsilon)}, \quad D=4-2 \epsilon .
$$

The integrals are independent of $\mu$ in the limit $\epsilon \rightarrow 0$. We follow the conventions for momenta as stated in the manual of LoopTools-2.15 [45].

\section{References}

[1] ATLAS collaboration, G. Aad et al., Observation of a new particle in the search for the Standard Model Higgs boson with the ATLAS detector at the LHC, Phys. Lett. $\mathbf{B 7 1 6}$ (2012) $1[1207.7214]$.

[2] CMS collaboration, S. Chatrchyan et al., Observation of a New Boson at a Mass of 125 GeV with the CMS Experiment at the LHC, Phys. Lett. B716 (2012) 30 [1207.7235]. 
[3] CMS collaboration, V. Khachatryan et al., Constraints on the spin-parity and anomalous $H V V$ couplings of the Higgs boson in proton collisions at 7 and 8 TeV, Phys. Rev. D92 (2015) 012004 [1411.3441].

[4] ATLAS collaboration, G. Aad et al., Study of the spin and parity of the Higgs boson in diboson decays with the ATLAS detector, Eur. Phys. J. C75 (2015) 476 [1506. 05669].

[5] CMS collaboration, A. M. Sirunyan et al., Combined measurements of Higgs boson couplings in protonâĂŞproton collisions at $\sqrt{s}=13$ TeV, Eur. Phys. J. C79 (2019) 421 [1809.10733].

[6] ATLAS collaboration, Combined measurements of Higgs boson production and decay using up to $80 \mathrm{fb}^{-1}$ of proton-proton collision data at $\sqrt{\mathrm{s}}=13 \mathrm{TeV}$ collected with the ATLAS experiment, Tech. Rep. ATLAS-CONF-2019-005, CERN, Geneva, Mar, 2019.

[7] C.-Y. Chen, S. Dawson and I. M. Lewis, Exploring resonant di-Higgs boson production in the Higgs singlet model, Phys. Rev. D91 (2015) 035015 [1410.5488].

[8] P. Huang, A. Joglekar, B. Li and C. E. M. Wagner, Probing the Electroweak Phase Transition at the LHC, Phys. Rev. D93 (2016) 055049 [1512.00068].

[9] I. M. Lewis and M. Sullivan, Benchmarks for Double Higgs Production in the Singlet Extended Standard Model at the LHC, Phys. Rev. D96 (2017) 035037 [1701.08774].

[10] J. Alison et al., Higgs Boson Pair Production at Colliders: Status and Perspectives, in Double Higgs Production at Colliders Batavia, IL, USA, September 4, 2018-9, 2019 (B. Di Micco, M. Gouzevitch, J. Mazzitelli and C. Vernieri, eds.), 2019, 1910.00012, https://lss.fnal.gov/archive/2019/conf/fermilab-conf-19-468-e-t.pdf.

[11] B. Batell, M. McCullough, D. Stolarski and C. B. Verhaaren, Putting a Stop to di-Higgs Modifications, JHEP 09 (2015) 216 [1508.01208].

[12] S. Dawson, A. Ismail and I. Low, WhatâĂŹs in the loop? The anatomy of double Higgs production, Phys. Rev. D91 (2015) 115008 [1504.05596].

[13] P. Huang, A. Joglekar, M. Li and C. E. M. Wagner, Corrections to di-Higgs boson production with light stops and modified Higgs couplings, Phys. Rev. D97 (2018) 075001 [1711.05743].

[14] C.-Y. Chen, J. Kozaczuk and I. M. Lewis, Non-resonant Collider Signatures of a Singlet-Driven Electroweak Phase Transition, JHEP 08 (2017) 096 [1704.05844].

[15] P. Basler, S. Dawson, C. Englert and M. MÃijhlleitner, Di-Higgs Peaks and Top Valleys: Interference Effects in Higgs Sector Extensions, 1909.09987.

[16] M. Carena, Z. Liu and M. Riembau, Probing the electroweak phase transition via enhanced di-Higgs boson production, Phys. Rev. D97 (2018) 095032 [1801.00794].

[17] S. Heinemeyer, W. Hollik and G. Weiglein, FeynHiggs: A Program for the calculation of the masses of the neutral CP even Higgs bosons in the MSSM, Comput. Phys. Commun. 124 (2000) 76 [hep-ph/9812320].

[18] S. Heinemeyer, W. Hollik and G. Weiglein, The Masses of the neutral CP - even Higgs bosons in the MSSM: Accurate analysis at the two loop level, Eur. Phys. J. C9 (1999) 343 [hep-ph/9812472].

[19] G. Degrassi, S. Heinemeyer, W. Hollik, P. Slavich and G. Weiglein, Towards high precision predictions for the MSSM Higgs sector, Eur. Phys. J. C28 (2003) 133 [hep-ph/0212020].

[20] M. Frank, T. Hahn, S. Heinemeyer, W. Hollik, H. Rzehak and G. Weiglein, The Higgs Boson 
Masses and Mixings of the Complex MSSM in the Feynman-Diagrammatic Approach, JHEP 02 (2007) 047 [hep-ph/0611326].

[21] T. Hahn, S. Heinemeyer, W. Hollik, H. Rzehak and G. Weiglein, High-Precision Predictions for the Light CP -Even Higgs Boson Mass of the Minimal Supersymmetric Standard Model, Phys. Rev. Lett. 112 (2014) 141801 [1312.4937].

[22] H. Bahl and W. Hollik, Precise prediction for the light MSSM Higgs boson mass combining effective field theory and fixed-order calculations, Eur. Phys. J. C76 (2016) 499 [1608.01880].

[23] H. Bahl, S. Heinemeyer, W. Hollik and G. Weiglein, Reconciling EFT and hybrid calculations of the light MSSM Higgs-boson mass, Eur. Phys. J. C78 (2018) 57 [1706. 00346].

[24] J. M. Campbell, J. W. Huston and W. J. Stirling, Hard Interactions of Quarks and Gluons: A Primer for LHC Physics, Rept. Prog. Phys. 70 (2007) 89 [hep-ph/0611148].

[25] U. Ellwanger, C. Hugonie and A. M. Teixeira, The Next-to-Minimal Supersymmetric Standard Model, Phys. Rept. 496 (2010) 1 [0910.1785].

[26] U. Ellwanger, J. F. Gunion and C. Hugonie, NMHDECAY: A Fortran code for the Higgs masses, couplings and decay widths in the NMSSM, JHEP 02 (2005) 066 [hep-ph/0406215].

[27] U. Ellwanger and C. Hugonie, NMHDECAY 2.0: An Updated program for sparticle masses, Higgs masses, couplings and decay widths in the NMSSM, Comput. Phys. Commun. 175 (2006) 290 [hep-ph/0508022].

[28] G. Degrassi and P. Slavich, On the radiative corrections to the neutral Higgs boson masses in the NMSSM, Nucl. Phys. B825 (2010) 119 [0907.4682].

[29] A. Djouadi, J. Kalinowski and M. Spira, HDECAY: A Program for Higgs boson decays in the standard model and its supersymmetric extension, Comput. Phys. Commun. 108 (1998) 56 [hep-ph/9704448].

[30] F. Domingo and U. Ellwanger, Updated Constraints from B Physics on the MSSM and the NMSSM, JHEP 12 (2007) 090 [0710.3714].

[31] G. Belanger, F. Boudjema, C. Hugonie, A. Pukhov and A. Semenov, Relic density of dark matter in the NMSSM, JCAP 0509 (2005) 001 [hep-ph/0505142].

[32] ATLAS collaboration, Search for direct top squark pair production in the 3-body decay mode with a final state containing one lepton, jets, and missing transverse momentum in $\sqrt{s}=13 T e V$ pp collision data with the ATLAS detector, Tech. Rep. ATLAS-CONF-2019-017, CERN, Geneva, May, 2019.

[33] CMS collaboration, Search for direct top squark pair production in events with one lepton, jets and missing transverse energy at $13 \mathrm{TeV}$, Tech. Rep. CMS-PAS-SUS-19-009, CERN, Geneva, 2019.

[34] N. Blinov and D. E. Morrissey, Vacuum Stability and the MSSM Higgs Mass, JHEP 03 (2014) 106 [1310.4174].

[35] CMS collaboration, A. M. Sirunyan et al., Search for additional neutral MSSM Higgs bosons in the $\tau \tau$ final state in proton-proton collisions at $\sqrt{s}=13$ TeV, JHEP 09 (2018) 007 [1803.06553].

[36] ATLAS collaboration, M. Aaboud et al., Search for additional heavy neutral Higgs and gauge bosons in the ditau final state produced in $36 \mathrm{fb} b^{1}$ of pp collisions at $\sqrt{s}=13 \mathrm{TeV}$ with the ATLAS detector, JHEP 01 (2018) 055 [1709.07242]. 
[37] ATLAS collaboration, Prospects for the search for additional Higgs bosons in the ditau final state with the ATLAS detector at HL-LHC, Tech. Rep. ATL-PHYS-PUB-2018-050, CERN, Geneva, Dec, 2018.

[38] ATLAS collaboration, Projections for measurements of Higgs boson cross sections, branching ratios, coupling parameters and mass with the ATLAS detector at the HL-LHC, Tech. Rep. ATL-PHYS-PUB-2018-054, CERN, Geneva, Dec, 2018.

[39] P. Basler, S. Dawson, C. Englert and M. MÃijhlleitner, Showcasing HH production: Benchmarks for the LHC and HL-LHC, Phys. Rev. D99 (2019) 055048 [1812.03542].

[40] M. Carena, H. E. Haber, I. Low, N. R. Shah and C. E. M. Wagner, Alignment limit of the NMSSM Higgs sector, Phys. Rev. D93 (2016) 035013 [1510.09137].

[41] G. Belanger, F. Boudjema, A. Pukhov and A. Semenov, micrOMEGAs: Version 1.3, Comput. Phys. Commun. 174 (2006) 577 [hep-ph/0405253].

[42] G. Belanger, F. Boudjema, A. Pukhov and A. Semenov, MicrOMEGAs 2.0: A Program to calculate the relic density of dark matter in a generic model, Comput. Phys. Commun. 176 (2007) 367 [hep-ph/0607059].

[43] G. Belanger, F. Boudjema, A. Pukhov and A. Semenov, micrOMEGAs 3: A program for calculating dark matter observables, Comput. Phys. Commun. 185 (2014) 960 [1305.0237].

[44] Planck collaboration, N. Aghanim et al., Planck 2018 results. VI. Cosmological parameters, 1807.06209.

[45] T. Hahn and M. Perez-Victoria, Automatized one loop calculations in four-dimensions and D-dimensions, Comput. Phys. Commun. 118 (1999) 153 [hep-ph/9807565]. 